\title{
HODOGRAPH METHOD IN MHD ORTHOGONAL FLUID FLOWS
}

\section{P.V. NGUYEN and O.P. CHANDNA}

Department of Mathematics and Statistics

University of Windsor. Windsor. ()ntario

Canada N9B 3P4

(Received February 8, 1991)

\begin{abstract}
Equations for steady plane MHD orthogonal flows of a viscous incompressible thid of finite electrical conductivity are recast in the hodograph plane by using the Legendre transform function of the streanfuntion. Three examples are studied to illustrate the developed theory. Solutions and geometries for these examples are determined.
\end{abstract}

Key Words and Plirases: Steady. Hodographic study. orthogonal. Magnetohydrodynamic. electrically conducting. finite conductivity.

1080 AMS Subject Classification Code 76W05

1. Introduction. This paper deals with the application of the hodograph transformation for solving a system of non-linear partial differential equations governing steady plane. magnetohydrodynamic flow of a viscous incompressible fluid in the presence of a magnetic field. W. F. Ames [1] has given an excellent survey to this method together with its applications to various other fields. Recently. O. P. Chandua et al. [2.3] used the hodograph and Legendre transformations to study non-Newtonian steady plane aligned and transverse MHD flows. O. P. Chandna et al. [4] also applied this technique to Navier Stokes equations. In this paper we consider the magnetic and velocity field vector are mutually orthogonal and the electrical conductivity of the fluid is taken to be finite. Since electrical conductivity is finite for most viscons fluid. our accounting for finite electrical conductivity makes the flow problem realistic and attractive from both a mathematic and physical point of view. We study our flows with the objective of determining exact solutions to various flow configurations. The plan of this paper is as follows: In section 2 the equations are cast into a convenient form for this work. Section 3 contains the transformation of equations to the hodograph plane so that the role of independent variables $x$. $y$ and the dependent variables $u$. $v$ are interchanged. In section 4 we introduce a Legendre transform function of the streamfunction and obtain a system of three equations in the Legendre transform function and the proportionality function. In section 5. we demonstrate the use of theoretical results found in section 4 by determining solutions to the following flows: (a) vortex flows (b) radial flows (c) spiral flows

2. Basic equations. The steady. plane flow of a viscous. incompressible fluid of finite electrical conductivity is governed by the following system of equations:

$$
\begin{gathered}
\frac{\partial u}{\partial x}+\frac{\partial v}{\partial y}=0 \\
\zeta\left(u \frac{\partial u}{\partial y}+v \frac{\partial u}{\partial y}\right)+\frac{\partial p}{\partial x}=\mu\left(\frac{\partial^{2} u}{\partial x^{2}}+\frac{\partial^{2} u}{\partial y^{2}}\right)-\mu^{*} j H_{2} \\
\zeta\left(u \frac{\partial v}{\partial x}+v \frac{\partial v}{\partial y}\right)+\frac{\partial p}{\partial y}=\mu\left(\frac{\partial^{2} v}{\partial x^{2}}+\frac{\partial^{2} v}{\partial y^{2}}\right)+\mu^{*} j H_{1} \\
u H_{2}-v H_{1}=\frac{1}{\mu^{*} \sigma} j+K \\
\frac{\partial H_{1}}{\partial x}+\frac{\partial H_{2}}{\partial y}=0 \\
j=\frac{\partial H_{2}}{\partial x}-\frac{\partial H_{1}}{\partial y}
\end{gathered}
$$


where $u . v$ are the components of the velocity field $\mathrm{V} . H_{1} . H_{2}$ are the components of the magnetic field H. p the pressure. $\zeta$ the constant fluid density. $\mu$ the constant coefficient of viscosity and $\mu^{*}$ the constant magnetic permealility. Here $K$ is an arbitrary constant of integration obtained from the diffusion equation

$$
\operatorname{curl}\left[\mathbf{V} \times \mathbf{H}-\frac{1}{\mu^{*} \sigma} \operatorname{curl} \mathbf{H}\right]=\mathbf{0}
$$

Introducing the functions

$$
\omega=\frac{\partial v}{\partial x}-\frac{\partial u}{\partial y} \cdot h=\frac{1}{2} \zeta|\mathbf{V}|^{2}+p
$$

where

$$
|\mathbf{V}|^{2}=u^{2}+v^{2}
$$

to the above system of equations. we obtain the following system:

$$
\begin{gathered}
\frac{\partial u}{\partial x}+\frac{\partial v}{\partial y}=0 \\
\frac{\partial h}{\partial x}=\zeta v \omega-\mu \omega_{y}-\mu^{*} j H_{2} \\
\frac{\partial h}{\partial y}=-\zeta u \omega+\mu \omega_{x}+\mu^{*} j H_{1} \\
u H_{2}-v H_{1}=\frac{1}{\mu^{*} \sigma} j+K \\
\frac{\partial H_{1}}{\partial x}+\frac{\partial H_{2}}{\partial y}=0 \\
j=\frac{\partial H_{2}}{\partial x}-\frac{\partial H_{1}}{\partial y} \\
\omega=\frac{\partial v}{\partial x}-\frac{\partial u}{\partial y}
\end{gathered}
$$

of seven partial differential equations in seven unknowns $u \cdot v \cdot H_{1}, H_{2}, \omega . j$ and $h$ as functions of $x . y$.

We consider our flows to be orthogonal flows. A plane flow is said to be orthogonal when the velocity field vector and the magnetic field vector are mutually perpendicular in the flow region. From this definition. we have

$$
\mathbf{H}=\mathbf{k} \times f(x, y) \mathbf{V}
$$

where $\mathbf{k}=(0.0 .1)$ and $f$ is a scalar function.

Using (2.15) in the system of equations (2.8) to (2.14). we get

$$
\begin{gathered}
\frac{\partial u}{\partial x}+\frac{\partial v}{\partial y}=0 \\
\frac{\partial h}{\partial x}=\zeta v w-\mu \omega_{y}-\mu^{*} j f u \\
\frac{\partial h}{\partial y}=-\zeta u w+\mu \omega_{x}-\mu^{*} j f v \\
f\left(u^{2}+v^{2}\right)=\frac{1}{\mu^{*} \sigma} j+K \\
-f \omega+u \frac{\partial f}{\partial y}-v \frac{\partial f}{\partial x}=0
\end{gathered}
$$




$$
\begin{aligned}
& j=u \frac{\partial f}{\partial x}+" \frac{\partial f}{\partial y} \\
& \omega=\frac{\partial u}{\partial x}-\frac{\partial u}{\partial y}
\end{aligned}
$$

3. Equations in the Hodograph Plane. Letting the flow variables $u=u(x \cdot y)) \cdot v=$ $v(x, y)$ to be such that. in the region of flow under consideration. the Jacobian

$$
J=\frac{\partial(u \cdot v)}{\partial(x . y)}=u_{x} v_{y}-u_{y} v_{x} \neq 0 .|J|<x
$$

we may consider $x . y$ as functions of $u . v$ By means of

$$
x=x(u . v) . \quad y=y(u . v)
$$

we derive the following relations

$$
\left.\begin{array}{c}
\frac{\partial u}{\partial x}=J \frac{\partial y}{\partial v} . \quad \frac{\partial u}{\partial y}=-J \frac{\partial x}{\partial v} \\
\frac{\partial v}{\partial x}=-J \frac{\partial y}{\partial u} . \quad \frac{\partial v}{\partial y}=J \frac{\partial x}{\partial u}
\end{array}\right\}
$$
tion.

Employing these transformation relations for the first order partial derivatives appearing in the system of equations (2.16) to (2.22). weobtain the system of equations in the $(u, v)$ - plane as follows:

where

$$
\begin{gathered}
\frac{\partial x}{\partial u}+\frac{\partial y}{\partial v}=0 \\
\bar{J} \frac{\partial(\bar{h} \cdot y)}{\partial(u \cdot v)}=\zeta v \bar{\omega}-\mu \bar{J} W_{1}-\mu^{*} \bar{j} \bar{f} u \\
\bar{J} \frac{\partial(x \cdot \bar{h})}{\partial(u \cdot v)}=-\zeta u \bar{\omega}+\mu \bar{J} W_{2}-\mu^{*} \bar{j} \bar{f} v \\
\bar{f}\left(u^{2}+v^{2}\right)=\frac{1}{\mu^{*} \sigma} \bar{j}+K \\
-\bar{f} \bar{\omega}+\bar{J}\left[u \frac{\partial(x \cdot \bar{f})}{\partial(u \cdot v)}-v \frac{\partial(\bar{f} \cdot y)}{\partial(u \cdot v)}\right]=0 \\
\bar{j}=\bar{J}\left[u \frac{\partial(\bar{f} \cdot y)}{\partial(u \cdot v)}+v \frac{\partial(x \cdot \bar{f})}{\partial(u \cdot v)}\right] \\
\bar{\omega}=\bar{J}\left[\frac{\partial x}{\partial v}-\frac{\partial y}{\partial u}\right]
\end{gathered}
$$

$$
\bar{J}=\left[\frac{\partial(x \cdot y)}{\partial(u \cdot v)}\right]^{-1} \cdot W_{1}=\frac{\partial(x \cdot \bar{\omega})}{\partial(u \cdot v)} \cdot W_{2}=\frac{\partial(\bar{\omega} \cdot y)}{\partial(u \cdot v)}
$$

This is a system of seven partial differential equations in six unknown functions $x=$ $x(u, v) \cdot y=y(u, v) \cdot \bar{\omega}=\bar{\omega}(u, v) \cdot \bar{f}=\bar{f}(u \cdot v) \cdot \bar{j}=\bar{j}(u, v)$ and $\bar{h}=\bar{h}(u, v)$ and an arbitrary constant $K$. Once $x, y \cdot \bar{\omega} \cdot \bar{f}, \bar{j}$ and $\bar{h}$ are deternined. we can find $u=u(x, y) \cdot v=$ $v(x, y) . \omega=\omega(x, y) . f=f(x, y) . j=j(x, y)$ and $h=h(x, y)$ which are the solutions for the system of equations (2.16) to (2.22). 
Equations in Legendre transform function and $\bar{f}(u . v)$.

The equation of continuity implies the existence of a strcamfunction $\psi(f, y)$. so that

$$
d \psi=-v d x+u d y \text { or } \frac{\partial \psi}{\partial x}=-v \cdot \frac{\partial \psi}{\partial y}=u
$$

Likewise. equation (3.4) implies the existence of a function $L(u . v)$. called the Legendre transform function of the streamfunction $\psi(x, y)$. so that

$$
d L=-y d u+x d v \text { or } \frac{\partial L}{\partial u}=-y \cdot \frac{\partial L}{\partial v}=x
$$

and the two functions $\psi(x, y)$ and $L(u, v)$ are related by

$$
L(u . v)=v x-u y+\psi(x . y)
$$

Introducing $L(u, v)$ into the system of equations (3.4) to (3.10). we obtain

$$
\begin{gathered}
\bar{J} \frac{\partial\left(\frac{\partial L}{\partial u} \cdot \bar{h}\right)}{\partial(u \cdot v)}=\zeta v \bar{\omega}-\mu \bar{J} W_{1}-\mu^{*} \bar{j} \bar{f} u \\
\bar{J} \frac{\partial\left(\frac{\partial L}{\partial v} \cdot \bar{h}\right)}{\partial(u \cdot v)}=-\zeta u \bar{\omega}+\mu \bar{J} W_{2}-\mu^{*} \bar{j} \bar{f} v \\
\bar{f}\left(u^{2}+v^{2}\right)=\frac{1}{\mu^{*} \sigma} \bar{j}+K \\
-\bar{f} \bar{\omega}+\bar{J}\left[u \frac{\partial\left(\frac{\partial L}{\partial v \cdot} \cdot \bar{f}\right)}{\partial(u \cdot v)}-v \frac{\partial\left(\frac{\partial L}{\partial u} \cdot \bar{f}\right)}{\partial(u \cdot v)}\right]=0 \\
\bar{j}=\bar{J}\left[u \frac{\partial\left(\frac{\partial L}{\partial u} \cdot \bar{f}\right)}{\partial(u \cdot v)}+v \frac{\partial\left(\frac{\partial L}{\partial v} \cdot \bar{f}\right)}{\partial(u \cdot v)}\right] \\
\bar{\omega}=\bar{J}\left[\frac{\partial^{2} L}{\partial v^{2}}+\frac{\partial^{2} L}{\partial u^{2}}\right]
\end{gathered}
$$

where

$$
\begin{aligned}
& \bar{J}=\left[\frac{\partial^{2} L}{\partial v^{2}} \frac{\partial^{2} L}{\partial u^{2}}-\left(\frac{\partial^{2} L}{\partial u \partial v}\right)^{2}\right]^{-1} \\
& W_{1}=\frac{\partial\left(\frac{\partial L}{\partial v} \cdot \bar{\omega}\right)}{\partial(u \cdot v)} \cdot W_{2}=\frac{\partial\left(\frac{\partial L}{\partial u} \cdot \bar{\omega}\right)}{\partial(u \cdot v)}
\end{aligned}
$$

By using the integrability condition

$$
\begin{aligned}
& {\left[\bar{J} \frac{\partial^{2} L}{\partial u \partial v} \frac{\partial}{\partial v}-\bar{J} \frac{\partial^{2} L}{\partial v^{2}} \frac{\partial}{\partial u}\right]\left(\bar{J} \frac{\partial\left(\frac{\partial L}{\partial u} \cdot \bar{h}\right)}{\partial(u \cdot v}\right) } \\
= & {\left[\bar{J} \frac{\partial^{2} L}{\partial u^{2}} \frac{\partial}{\partial v}-\bar{J} \frac{\partial^{2} L}{\partial u \partial v} \frac{\partial}{\partial u}\right]\left(\bar{J} \frac{\partial\left(\frac{\partial L}{\partial v} \cdot \bar{h}\right)}{\partial(u \cdot v)}\right) }
\end{aligned}
$$

we eliminate $\bar{h}($ u.v) from equations (3.15) and (3.16) to obtain

$$
\begin{gathered}
\zeta\left(u W_{2}+v W_{1}\right)-\mu\left[\frac{\partial\left(\frac{\partial L}{\partial v} \cdot \bar{J} W_{1}\right)}{\partial(u \cdot v)}+\frac{\partial\left(\frac{\partial L}{\partial u} \cdot \bar{J} W_{2}\right)}{\partial(u \cdot v)}\right] \\
+\mu^{*}\left[v \frac{\partial\left(\frac{\partial L}{\partial u} \cdot \overline{f j}\right)}{\partial(u \cdot v)}-u \frac{\partial\left(\frac{\partial L}{\partial v} \cdot \overline{f j}\right)}{\partial(u \cdot v)}+\overline{f j}\left(\frac{\partial^{2} L}{\partial u^{2}}+\frac{\partial^{2} L}{\partial v^{2}}\right)\right]=0
\end{gathered}
$$


Employing (3.17) in (3.23) and making use of (3.18). we get

$$
\begin{gathered}
\zeta\left(u W_{2}+u W_{1}\right)-\mu\left[\frac{\partial\left(\frac{\partial L}{\partial v} \cdot \bar{J} W_{1}\right)}{\partial(u \cdot v)}+\frac{\partial\left(\frac{\partial L}{\partial u} \cdot \bar{J} W_{2}\right)}{\partial(u \cdot v)}\right] \\
+\mu^{*^{2}} \sigma \bar{f}\left[v \frac{\partial\left(\frac{\partial L}{\partial u} \cdot\left(u^{2}+v^{2}\right) \bar{f}\right)}{\partial(u \cdot v)}-u \frac{\partial\left(\frac{\partial L}{\partial v} \cdot\left(u^{2}+v^{2} \bar{f}\right)\right.}{\partial(u \cdot v)}\right]=0
\end{gathered}
$$

From (3.17) and (3.19). we olbtain

$$
\mu^{*} \sigma\left(u^{2}+v^{2}\right) \bar{f}-\bar{J}\left[u \frac{\partial\left(\frac{\partial L}{\partial u} \cdot \bar{f}\right)}{\partial(u \cdot v)}+v \frac{\partial\left(\frac{\partial L}{\partial v} \cdot \bar{f}\right)}{\partial(u \cdot v)}\right]=\mu^{*} \sigma K
$$

In summary. we have the following theorem:

Theorem 1. If $L(u . v)$ is the Legendre transform function of a streamfunction of steady plane orthogonal flow of an incompressible viscous fluid of finite electrical conductivity and $\bar{f}(u, v)$ is the transformed proportionality function. then $L(u, v)$ and $\bar{f}(u, v)$ must satisfy equations (3.18). (3.24) and (3.25) where $\bar{\omega} . \bar{J} . W_{1} . W_{2}$ and $\bar{j}$ are given by (3.10). (3.21). (3.22) and (3.17).

Once a solution $\{L(u, v) . \bar{f}(u . v)\}$ is found. for which $\bar{J}$ evaluated from (3.21) satisfies $0<|J|<x$. the solution for the velocity components are obtained by solving equations (3.13) simultaneously. Having obtained the velocity components $u=u(x, y) . \quad v=v(x, y)$. we obtain $f(x, y)$ in the physical plane from the solution for $\bar{f}(u, v)$ in the hodograph plane. Using $V(x, y)$ and $f(x, y)$ in (2.6). (2.7). (2.9) and (2.10). we determine other flow variables in the physical plane.

We now develop the results of the above theorem in polar coordinates $(q . \theta)$ in the hodograph plane. Defining

$$
q^{2}=u^{2}+v^{2} \cdot \theta=\tan ^{-1}\left(\frac{v}{u}\right)
$$

we have the following transformation relations

$$
\begin{gathered}
\frac{\partial}{\partial u}=\cos \theta \frac{\partial}{\partial q}-\frac{\sin \theta}{q} \frac{\partial}{\partial \theta} \\
\frac{\partial}{\partial v}=\sin \theta \frac{\partial}{\partial q}+\frac{\cos \theta}{q} \frac{\partial}{\partial \theta} \\
\frac{\partial(F \cdot G)}{\partial(u \cdot v)}=\frac{\partial\left(F^{*} \cdot G^{*}\right)}{\partial(q \cdot \theta)} \frac{\partial(q \cdot \theta)}{\partial(u \cdot v)}=\frac{1}{q} \frac{\partial\left(F^{*} \cdot G^{*}\right)}{\partial(q \cdot \theta)}
\end{gathered}
$$

where $F(u, v)=F^{*}(q . \theta), G(u, v)=G^{*}(q, \theta)$ are continuously differentiable functions.

Letting $L^{*}(q, \theta), f^{*}(q, \theta), j^{*}(q, \theta), J^{*}(q, \theta)$ and $\omega^{*}(q, \theta)$ to be respectively the transformed functions of $L(u, v)$. $\quad \bar{f}(u, v), \bar{j}(u, v) . \bar{J}(u, v)$ and $\bar{\omega}(u, v)$ in $(q, \theta)$ - coordinates. and using the above transformation relations in the equations of theorem 1 . we obtain the following results:

Corollary. If $L^{*}(q . \theta)$ and $f^{*}(q . \theta)$ are the Legendre transform functions of a streamfunction and the proportionality function respectively of the equations governing the motion of steady plane orthogonal flow of an incompressible viscous fluid of finite electrical conductivity, then $L^{*}(q . \theta)$ and $f^{*}(q . \theta)$ must satisfy equations: 


$$
\begin{aligned}
& \zeta q\left[\cos \theta W_{2}^{*}+\sin \theta W_{1}^{*}\right]-\frac{\mu}{q}\left[\frac{\partial\left(\sin \theta \frac{\partial L^{*}}{\partial q}+\frac{\cos \theta}{q} \frac{\partial L^{*}}{\partial \theta} \cdot J^{*} W_{1}^{*}\right)}{\partial(q \cdot \theta)}\right. \\
& \left.+\frac{\partial\left(\cos \theta \frac{\partial L^{*}}{\partial q}-\frac{\sin \theta}{q} \frac{\partial L^{*}}{\partial \theta^{*}} \cdot J^{*} W_{2}^{*}\right)}{\partial(q \cdot \theta)}\right] \\
& +\mu^{* 2} \sigma f^{*}\left[\sin \theta \frac{\partial\left(\cos \theta \frac{\partial L^{*}}{\partial q}-\frac{\sin \theta}{q} \frac{\partial L^{*}}{\partial \theta} \cdot Q^{2} F^{*}\right)}{\partial(q \cdot \theta)}\right. \\
& \left.-\cos \theta \frac{\partial\left(\sin \theta \frac{\partial L^{*}}{\partial q}+\frac{\cos \theta}{q} \frac{\partial L_{*}}{\partial \theta} \cdot q^{2} f^{*}\right)}{\partial(q \cdot \theta)}\right]=0 \\
& f^{*} \omega^{*}-J^{*}\left[\cos \theta \frac{\partial\left(\sin \theta \frac{\partial L^{*}}{\partial q}+\frac{\cos \theta}{q} \frac{\partial L^{*}}{\partial \theta} \cdot f^{*}\right)}{\partial(q \cdot \theta)}\right. \\
& \left.-\sin \theta \frac{\partial\left(\cos \theta \frac{\partial L^{*}}{\partial q}-\frac{\sin \theta}{q} \frac{\partial L^{*}}{\partial \theta} \cdot f^{*}\right)}{\partial(q \cdot \theta)}\right]=0 \\
& \mu^{*} \sigma f^{*} q^{2}-J^{*}\left[\cos \theta \frac{\partial\left(\cos \theta \frac{\partial L^{*}}{\partial q}-\frac{\sin \theta}{q} \frac{\partial L^{*}}{\partial \theta} \cdot f^{*}\right)}{\partial(q \cdot \theta)}\right. \\
& \left.+\sin \theta \frac{\partial\left(\sin \theta \frac{\partial L^{*}}{\partial q}+\frac{\cos \theta}{q} \frac{\partial L^{*}}{\partial \theta} \cdot f^{*}\right)}{\partial(q \cdot \theta)}\right]=\mu^{*} \sigma K
\end{aligned}
$$

where

$$
\begin{gathered}
J^{*}(q . \theta)=q^{*}\left[q^{2} \frac{\partial^{2} L}{\partial q^{2}}\left\{q \frac{\partial L^{*}}{\partial q}+\frac{\partial^{2} L^{*}}{\partial \theta^{2}}\right\}-\left\{\frac{\partial L^{*}}{\partial \theta}-q \frac{\partial^{2} L^{*}}{\partial q \partial \theta}\right\}^{2}\right]^{-1} \\
\omega^{*}(q . \theta)=J^{*}\left[\frac{\partial^{2} L^{*}}{\partial q^{2}}+\frac{1}{q^{2}} \frac{\partial^{2} L^{*}}{\partial \theta^{2}}+\frac{1}{q} \frac{\partial L^{*}}{\partial q}\right] \\
W_{1}^{*}(q . \theta)=\frac{1}{q}\left[\frac{\partial\left(\sin \theta \frac{\partial L^{*}}{\partial q}+\frac{\cos \theta}{q} \frac{\partial L^{*}}{\partial \theta}, \omega^{*}\right)}{\partial(q . \theta)}\right] \\
W_{2}^{*}(q . \theta)=\frac{1}{q}\left[\frac{\partial\left(\cos \theta \frac{\partial L^{*}}{\partial q}-\frac{\sin \theta}{q} \frac{\partial L^{*}}{\partial \theta} \cdot \omega^{*}\right)}{\partial(q . \theta)}\right] \\
j^{*}=\mu^{*} \sigma\left(f^{*} q^{2}-K\right)
\end{gathered}
$$

Once a solution $\left\{L^{*}(q \cdot \theta) . f^{*}(q \cdot \theta)\right\}$ is known. we employ the relations

$$
\begin{aligned}
& x=\sin \theta \frac{\partial L^{*}}{\partial q}+\frac{\cos \theta}{q} \frac{\partial L^{*}}{\partial \theta} \\
& y=\frac{\sin \theta}{q} \frac{\partial L^{*}}{\partial \theta}-\cos \theta \frac{\partial L^{*}}{\partial q}
\end{aligned}
$$

to obtain the velocity components in the physical plane. Having obtained $u=u(x, y) . v=$ $v(x, y)$. we get $f(x, y)$ in the $(x, y)$ - plane from $f^{*}(q, \theta)$. The other flow variables are then determined by using the flow equations in the physical plane. 
4. Applications. In this section we study varions flow problems as applications of theorem 1 and its corollary.

Application 1. (Vortex flow) Let

$$
L^{*}(q \cdot \theta)=F(q) \cdot F^{\prime}(q) \neq 0 . F^{\prime \prime}(q) \neq 0
$$

be the Legendre transform function. Using (4.1) to equations (3.29) to (3.32). we get

$$
\begin{gathered}
J^{*}=\frac{q}{F^{\prime}(q) F^{\prime \prime}(q)} \cdot \omega^{*}(q)=\frac{q F^{\prime \prime}(q)+F^{\prime}(q)}{F^{\prime}(q) F^{\prime \prime}(q)} \\
W_{1}^{*}=-\frac{1}{q} \omega^{*^{\prime}}(q) \cos \theta F^{\prime}(q) \cdot W_{2}^{*}=\frac{1}{q} \omega^{*^{\prime}}(q) \sin \theta F^{\prime}(q)
\end{gathered}
$$

where prime denotes differentiation with respect to $q$.

Employing (4.2) and (4.3) in (3.26). (3.27) and (3.28). we obtain respectively

$$
\begin{gathered}
\frac{\mu}{q}\left\{\sin \theta\left[F^{\prime \prime}(q)\left(J^{*} W_{1}^{*}\right)_{\theta}+F^{\prime}(q)\left(J^{*} W_{2}^{*}\right)_{q}\right]\right. \\
\left.+\cos \theta\left[F^{\prime \prime}(q)\left(J^{*} W_{2}^{*}\right)_{\theta}-F^{\prime}(q)\left(J^{*} W_{1}^{*}\right)_{q}\right]\right\} \\
-\mu^{* 2} \sigma f^{*} F^{\prime}(q) \frac{\partial\left(q^{2} f^{*}\right)}{\partial q}=0 \\
\frac{\partial f^{*}}{\partial q}+\left[\frac{F^{\prime \prime}(q)}{F^{\prime}(q)}+\frac{1}{q}\right] f^{*}=0 \\
\frac{\partial f^{*}}{\partial \theta}-\mu^{*} \sigma q F^{\prime}(q) f^{*}=-\mu^{*} \sigma K \frac{F^{\prime}(q)}{q}
\end{gathered}
$$

A general solution for (4.5) is

$$
f^{*}(q, \theta)=\frac{\phi(\theta)}{q F^{\prime}(q)}
$$

where $\phi(\theta)$ is an arbitrary function of $\theta$.

Using (4.7) in (4.6). we get

$$
\frac{1}{F^{\prime}\left(q^{2}\right)} \frac{\phi^{\prime}(\theta)}{\phi(\theta)}+\frac{\mu^{*} \sigma K}{\phi(\theta)}=\mu^{*} \sigma \frac{q}{F^{\prime}(q)}
$$

Differentiating (4.8) with respect to $\theta$. we obtain

$$
\phi^{\prime \prime}(\theta) \phi(\theta)-\phi^{\prime 2}(\theta)-\mu^{*} \sigma K F^{\prime 2}(q) \phi^{\prime}(\theta)=0
$$

We consider two cases: $\phi^{\prime}(\theta)=0$ and $\phi^{\prime}(\theta) \neq 0$.

(i) $\underline{\phi^{\prime}}(\theta) \neq 0$

Equation (66) can be written as

$$
\frac{\phi^{\prime \prime}(\theta) \phi(\theta)-\phi^{\prime 2}(\theta)}{\phi^{\prime}(\theta)}=\mu^{*} \sigma K F^{\prime 2}(q)
$$

Equation (4.10) implies that

$$
\begin{gathered}
\frac{\phi^{\prime \prime}(\theta) \phi(\theta)-\phi^{\prime 2}(\theta)}{\phi^{\prime}(\theta)}=C \\
\mu^{*} \sigma K F^{\prime 2}(q)=C
\end{gathered}
$$

where $C$ is an arbitrary constaut. 
Since $F^{\prime \prime}(q) \neq 0$. it follows from equation (1.12) that

$$
K=0 \quad \text { and } \quad C=0
$$

Hence, equation (4.11) reduces to

$$
\frac{\phi^{\prime \prime}(\theta)}{\phi^{\prime}(\theta)}-\frac{\phi^{\prime}(\theta)}{\phi(\theta)}=0
$$

Integrating (4.14) twice with respect to $\theta$. we get

$$
\phi(\theta)=D_{2} \exp \left[D_{1} \theta\right]
$$

where $D_{1}$ and $D_{2}$ are nonzero arbitrary constants.

Using (4.13) and (4.15) in (4.8). we olbtain

$$
F^{\prime}(q)=\frac{D_{1}}{\mu^{*} \sigma} \frac{1}{q}
$$

Integrating (4.16) with respect to $q$. we have

$$
F(q)=\frac{D_{1}}{\mu^{*} \sigma} \ln q+D_{3}
$$

where $D_{3}$ is an arbitrary constant.

Substituting (4.15) and (4.16) in (4.7). we obtain

$$
f^{*}(\theta)=\frac{\mu^{*} \sigma D_{2}}{D_{1}} \quad \exp \left[D_{1} \theta\right]
$$

Employing (4.18) in the expression for $\omega^{*}(q)$ given in (4.2). we get

$$
\omega^{*}(q)=0
$$

Using (4.16). (4.18) and (4.19) in the equation (4.4). we obtain

$$
\frac{2 \mu^{* 3} \sigma^{2} D_{2}^{2}}{D_{1}^{2}} \exp \left(2 D_{1} \theta\right)=0
$$

which is impossible since the left hand side of the above equation is greater than zero.

Therefore. $L^{*}(q)=\frac{D_{1}}{\mu^{*} \sigma} \ln q+D_{3}$ is not the Legendre transform function of the streamfunction of the flow.

(ii) $\underline{\phi}(\theta)=0$

In this case. we have

$$
\phi(\theta)=D_{4}=\text { constant }
$$

Employing (4.20) in (4.8). we get

$$
F^{\prime}(q)=\frac{D_{t}}{K} q . \quad K \neq 0
$$

Hence.

$$
F(q)=\frac{D_{4}}{2 K} \quad q^{2}+D_{5}
$$

From (4.2). (4.7). (4.20) and (4.22). we obtain

$$
\begin{gathered}
f^{*}(q)=\frac{K}{q^{2}} \\
\omega^{*}=\frac{2 K}{D_{4}}
\end{gathered}
$$


Using (4.21). (4.23) and (4.24) in equation (4.4). we find that equation (4.4) is identically satisfied. Therefore.

$$
L^{*}(q . \theta)=F(q)=\frac{D_{4}}{2 K} q^{2}+D_{3} . K \neq 0
$$

is a Legendre transform function of the flow for which $f^{*}(q)$ is given by (4.23).

Writing (4.25) in the (u.v) - plane. we have

$$
L(u . u)=\frac{D_{t}}{2 K}\left(u^{2}+v^{2}\right)+D_{j}
$$

Employing (4.26) in (3.13). we obtain

$$
\mathrm{V}(x . y)=\frac{K}{D_{4}}(-y \cdot x)
$$

From (4.23) and (4.27). we determine

$$
\begin{gathered}
f(x, y)=\frac{D_{\ddagger}^{2}}{K\left(x^{2}+y^{2}\right)} \\
\mathbf{H}(x, y)=f(x . y)(-v \cdot u)=\frac{-D_{4}}{x^{2}+y^{2}}(x, y)
\end{gathered}
$$

Using (4.24). (4.27). (4.28) in equations (2.9) and (2.10). and integrating. we obtain

$$
h(x . y)=\frac{\zeta K^{2}}{D_{4}^{2}}\left(x^{2}+y^{2}\right)+D_{6}
$$

where $D_{6}$ is an arbitrary constant.

From (2.7). we determine the pressure to be

$$
p(x . y)=\frac{\zeta K^{2}}{2 D_{\ddagger}^{2}}\left(x^{2}+y^{2}\right)+D_{6}
$$

Summing up, we have the following theorem:

Theorem 2. If $L^{*}(q, \theta)=F(q)$ is the Legendre transform function of streamfunction for a steady, plane. orthogonal flow of an incompressible viscous fluid of finite electrical conductivity. then the flow in the physical plane is a vortex flow given by equations (4.27), (4.28) to (4.30).

\section{Application 2. (Radial flow)}

We let

$$
L^{*}(q, \theta)=A \theta+B \cdot A \neq 0
$$

to be the Legendre transform funciton. where $A$ and $B$ are arbitrary constants.

Evaluating $J^{*} \cdot \omega^{*}, W_{1}^{*}$ and $W_{2}^{*}$ as before. we get

$$
J^{*}=-\frac{q^{4}}{A^{2}} \cdot \omega^{*}=W_{1}^{*}=W_{2}^{*}=0
$$

Following the same analysis as in previous applications. we obtain the system of equations

$$
\begin{gathered}
\frac{\partial f^{*}}{\partial \theta}=0 \\
\frac{\partial f^{*}}{\partial q}+\frac{\mu^{*} \sigma A}{q} f^{*}=\frac{\mu^{*} \sigma K A}{q^{3}}
\end{gathered}
$$

Equations (4.33) yields

$$
f^{*}=f^{*}(q)
$$


Therefore. equation (4.34) becomes an ordinary differential equation which has the general solutions:

(i) $\underline{\mu^{*} \sigma A \neq 2}$

$$
f^{*}(q)=\frac{\mu^{*} \sigma K A}{\mu^{*} \sigma A-2} \quad q^{-2}+E_{1} q^{-\mu^{*} \sigma \cdot t}
$$

(ii) $\mu^{*} \sigma A=2$

$$
f^{*}(q)=\frac{2 K}{q^{2}} \ell n q+\frac{E_{2}}{q^{2}}
$$

where $E_{1}$ and $E_{2}$ are arbitrary constants.

Proceeding as before. we obtain the following theorem:

Theorem 3. If $L^{*}(q . \theta)=A \theta+B$ is the Legendre transform function of strcamfunction for a steady. plane. orthogonal flow of a viscons incompressible fluid of finite electrical conductivity. then the flow in the physical plane is a radial flow given by equations:

(i) $\quad \underline{A \neq 2 / \mu^{*} \sigma}$

$$
\begin{gathered}
\mathrm{V}(x \cdot y)=\left(\frac{A x}{x^{2}+y^{2}} \cdot \frac{A y}{x^{2}+y^{2}}\right) \\
\omega=0 \\
f(x \cdot y)=\frac{\mu^{*} \sigma K A}{\mu^{*} \sigma A-2} q^{-2}+E_{1} q^{-\mu^{*} \sigma .}
\end{gathered}
$$

where

$$
\begin{gathered}
q^{2}=A^{2} /\left(x^{2}+y^{2}\right) \\
\mathbf{H}(x, y)=\left[\frac{\mu^{*} \sigma K A}{\mu^{*} \sigma A-2} q^{-2}+E_{1} q^{-\mu^{*} \sigma . t}\right]\left(\frac{-A y}{x^{2}+y^{2}} \cdot \frac{A x}{x^{2}+y^{2}}\right) \\
j(x, y)=\mu^{*} \sigma E_{1} q^{\left(2-\mu^{*} \sigma . t\right)}+\frac{2 \mu^{*} \sigma K}{\mu^{*} \sigma A-2} \\
p(x, y)=\mu^{*} \int j H_{2} d x+j H_{1} d y-\frac{1}{2} \zeta A^{2} /\left(x^{2}+y^{2}\right)
\end{gathered}
$$

where $j . H_{1}$ and $H_{2}$ are given by (4.38) and (4.39).

(ii) $\underline{A=2 / \mu^{*} \sigma}$

$$
\begin{gathered}
\mathrm{V}(x, y)=\frac{2}{\mu^{*} \sigma}\left(\frac{x}{x^{2}+y^{2}} \cdot \frac{y}{x^{2}+y^{2}}\right) \\
\omega=0 \\
f(x, y)=\frac{2 K}{q^{2}} \ell \cdot n q+\frac{E_{2}}{q^{2}}
\end{gathered}
$$

where

$$
\begin{gathered}
q^{2}=\frac{4}{\left(\mu^{*} \sigma\right)^{2}\left(x^{2}+y^{2}\right)} \\
\mathbf{H}(x, y)=\frac{2}{\mu^{*} \sigma}\left[\frac{2 K}{q^{2}} \ln q+\frac{E_{2}}{q^{2}}\right]\left(\frac{-y}{x^{2}+y^{2}} \cdot \frac{x}{x^{2}+y^{2}}\right) \\
j(x, y)=\mu^{*} \sigma K\left(\ln q^{2}-1\right)+\mu^{*} \sigma E_{2}
\end{gathered}
$$

and the presourc is given by (1.40) where now $j . H_{1}$ and $H_{2}$ are given lig (4.41) and (1.42).

Application 3. (Spiral flow)

Letting

$$
L^{*}(q . \theta)=G_{1} \ell n q+G_{2} \theta
$$

to be the Legendre transform function. where $G_{1}$ and $G_{2}$ are non-zero arbitrary constants.

Evaluating $J^{*} . \omega^{*} . W_{1}^{*}$ and $W_{2}^{*}$ as before. we get 


$$
J^{*}=\frac{-q^{4}}{G_{1}^{2}+G_{2}^{2}} \cdot \omega^{*}=W_{2}^{*}=W_{1}^{*}=0
$$

Proceeding as in previous applications. we obtain the following system of equations:

$$
\begin{gathered}
\frac{2 G_{1}}{q^{2}} f^{*}+\frac{G_{1}}{q} \frac{\partial f^{*}}{\partial q}+\frac{G_{2}}{q^{2}} \frac{\partial f^{*}}{\partial \theta}=0 \\
\frac{G_{1}}{q} \frac{\partial f^{*}}{\partial q}+\frac{G_{2}}{q^{2}} \frac{\partial f^{*}}{\partial \theta}=0 \\
\mu^{*} \sigma \quad q^{2} f^{*}+\frac{q^{2}}{G_{1}^{2}+G_{2}^{2}}\left[G_{2} q \frac{\partial f^{*}}{\partial q}-G_{1} \frac{\partial f^{*}}{\partial \theta}\right]=\mu^{*} \sigma K
\end{gathered}
$$

From (4.45) and (4.46). we get

$$
f^{*}=0
$$

which is a trivial solution for the system of equations (4.45) to (4.47). Therefore. the spiral flow is not possible.

\section{References}

1. W.F. Ames. Non-linear partial differential equation. Academic Press. New York. 1965.

2. P.V. Nguyen and O.P. Chandna. Hodographic study of non-Newtonian MHD aligned steady plane fluid flous. Internat. J. Math. \& Math. Sri. 13 ( 1990). 93-114.

3. O.P. Chandna and P.V. Nguyen. Hodograph method in non-Newtoniun MHD trunsverse fluid flows, J. of Engineering Mathematics 23 ( 1989). 119-139.

4. O.P. Chandna, R.M. Barron and A.C. Smith. Rotational plane flous of a viscous fluid. Siam J. Appl. Math. 42 ( 1982). 1323-1336. 


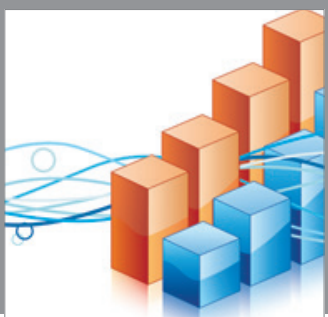

Advances in

Operations Research



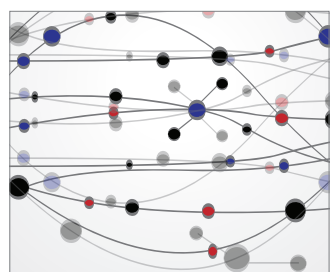

\section{The Scientific} World Journal
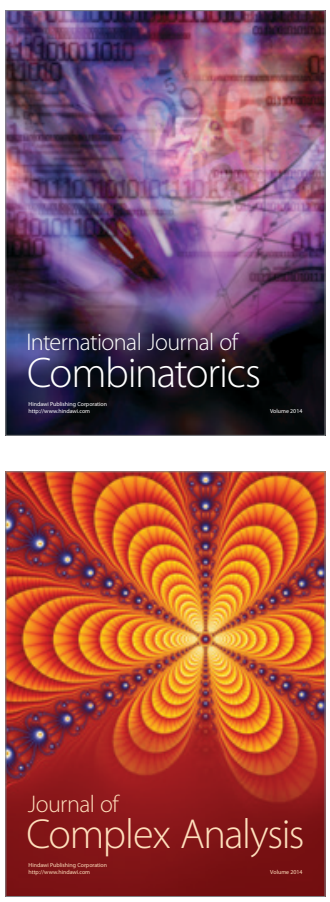

International Journal of

Mathematics and

Mathematical

Sciences
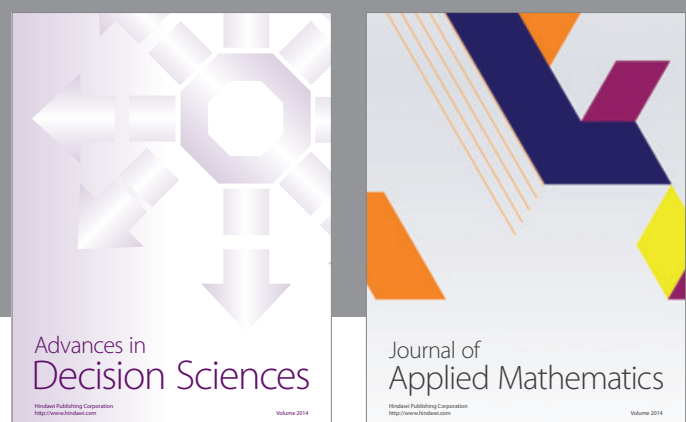

Journal of

Applied Mathematics
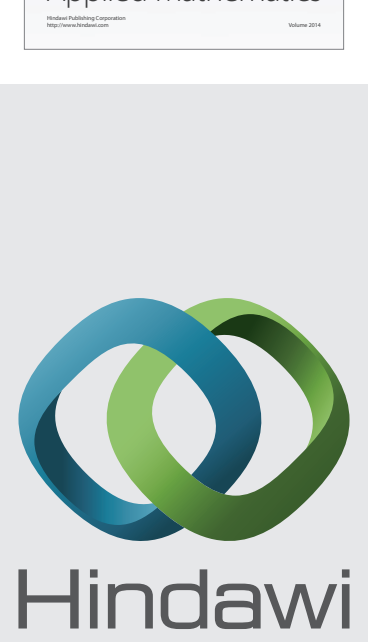

Submit your manuscripts at http://www.hindawi.com


Mathematical Problems in Engineering


Journal of

Function Spaces
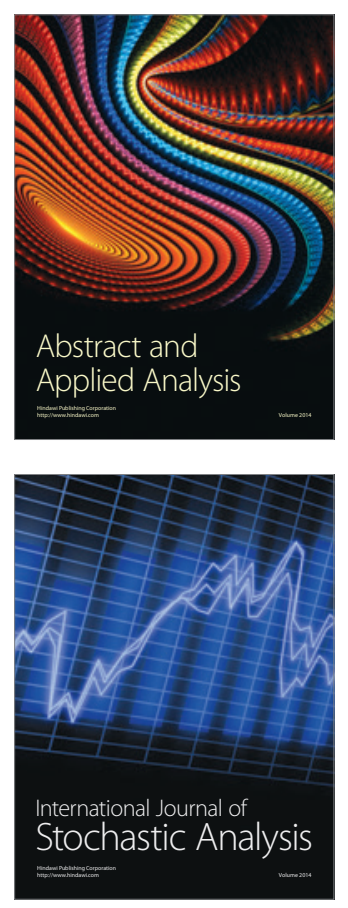

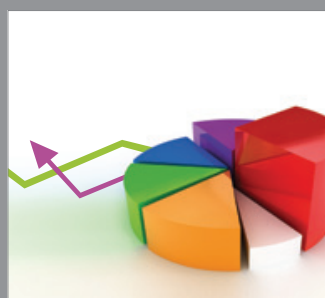

ournal of

Probability and Statistics

Promensencen
